\begin{tabular}{|c|l|}
\hline Title & Photoinduced structural changes in amorphous semiconductors \\
\hline Author(s) & Tanaka, K. \\
\hline Citation & $\begin{array}{l}\text { Semiconductors, 32(8), 861-866 } \\
\text { https://doi.org/10.1134/1187473 }\end{array}$ \\
\hline Issue Date & 1998-08 \\
\hline Doc URL & http://hdl.handle.net/2115/7410 \\
\hline Rights & Copyright $\odot$ 1998 A merican Institute of Physics \\
\hline Type & article \\
\hline File Information & Sc32_8.pdf () \\
\hline
\end{tabular}

Instructions for use 


\title{
Photoinduced structural changes in amorphous semiconductors
}

\author{
K. Tanaka
}

Department of Applied Physics, Faculty of Engineering, Hokkaido University, Sapporo 060, Japan

(Submitted February 16, 1998, accepted for publication February 23, 1998)

Fiz. Tekh. Poluprovodn. 32, 964-969 (August 1998)

A variety of photostructural changes observed in tetrahedral and chalcogenide amorphous semiconductors are reviewed from physical and chemical points of view. In particular, observations of the photodarkening and related phenomena in chalcogenide glasses are summarized, and structural models which have been proposed so far are criticized. (c) 1998 American Institute of Physics. [S1063-7826(98)01208-3]

\section{INTRODUCTION}

It is known that many substances exhibit structural changes when exposed to visible light. A well-known example in physical science may be the photographic reaction, in which migration of $\mathrm{Ag}^{+}$ions is induced in $\mathrm{Ag}$-halide crystals by photoelectronic excitation. ${ }^{1}$

Recently, it was found that amorphous semiconductors exhibit a variety of photoinduced phenomena. ${ }^{2-8}$ The following three features peculiar to amorphous semiconductors appear to be responsible for the observations: 1) valence electrons in semiconductors tend to be optically excited; 2) electron-lattice interaction appears to be strong in flexible networks; and 3) amorphous materials can possess a number of metastable structures.

The phenomena observed in amorphous semiconductors can be classified into two groups. One is bulk effects or photoinduced phenomena in single amorphous phases. ${ }^{3-8}$ The other is a kind of photochemical reaction such as the photodoping, the photoinduced oxidation, etc., for which the reader may refer to some publications. ${ }^{2,4,6,9}$

In the present paper we describe the bulk photoinduced phenomena. First, unified views common to tetrahedral and chalcogenide systems are pointed out and then characteristic differences are discussed. Second, the reversible photodarkening and related phenomena induced in chalcogenide glasses with illumination and annealing are considered.

\section{PROMISING OUTLOOK FOR BULK PHOTOINDUCED PHENOMENA}

\subsection{Observations}

As shown in Table I, the bulk photoinduced phenomena observed in tetrahedral and chalcogenide systems can be classified into some groups, depending on the densities of atomic sites involved. ${ }^{10}$

Photoinduced crystallization is the most dramatic phenomenon observed in both tetrahedral ${ }^{11}$ and chalcogenide amorphous semiconductors. ${ }^{12,13}$ The phenomena appear to be induced electronically ${ }^{13,14}$ and thermally,,$^{11,12}$ and the thermal process has been utilized as phase-change erasable memories. ${ }^{12}$. Clearly, the photocrystallization is irreversible, in the sense that the initial amorphous state cannot be recov- ered with annealing, ${ }^{1)}$ since the illuminated state is thermodynamically more stable (see, the inset in Table I).

The number of covalent bonds interchanged in the photo-crystallization processes seem to depend on the material of interest. For instance, in amorphous Se, which consists of distorted chain molecules, ${ }^{16,17}$ interchange of a few atomic bonds $(\sim 1 \%)$ seems to be sufficient to align the distorted chains into hexagonal crystals. ${ }^{2)}$ In contrast, in a ternary compound such as $\mathrm{Ge}-\mathrm{Sb}-\mathrm{Te}$ (Ref. 12), bond breakage and reconstruction of a greater number $(\sim 10 \%)$ may be needed to produce polycrystals, since compositional disorder is inherent in compound materials. In Table I, therefore, the atomic density contributing to the photocrystallization is represented roughly as $10^{22} \mathrm{~cm}^{-3}$. This phenomenon is understood to accompany structural changes in the long-range order, since crystals are produced.

Also observed in tetrahedral and chalcogenide systems are reversible phenomena involving sturctural changes at atomic sites of $10^{17}-10^{18} \mathrm{~cm}^{-3}$, approximately ppm order. ${ }^{5}$ The density is comparable to that of point defects in crystals, e.g., color centers in alkali halides, ${ }^{1}$ and it is far below a detection limit of experimental techniques such as $\mathrm{x}$-ray diffraction, which can provide direct structural information. Accordingly, mechanisms of these photoinduced phenomena are largely speculative, specifically, when related sites are ESR-inactive. The Staebler-Wronski effect, which refers to a degradation of photoconductive properties in amorphous hydrogenated $\mathrm{Si}$, is a reversible phenomenon which has been studied extensively. ${ }^{5}$ In chalcogenide glasses held at low temperatures $\left(\leqslant T_{g} / 2\right)$, illumination generates unpaired electron spins (photoinduced ESR), which accompany an increase in optical absorption (photoinduced mid-gap absorption) and decrease in the photoluminescence intensity (photoinduced photoluminescence fatigue). ${ }^{5}$ Common to all these phenomena is that dangling bonds are assumed to be photocreated. $^{s}$

Between the high " and low-density photoinduced phenomena described above, there exist several phenomena involving atomic sites of $\sim 1 \%$. $^{5}$ Interestingly, these kind of phenomena the demonstrated only in chalcogenide glasses. In tetrahedral materials, such photostructural changes seem to be still speculative. ${ }^{19,20}$ In these photoinduced phenomena in chalcogenide glasses, some are irrevers- 
TABLE I. A classification of bulk photoinduced phenomena observed in tetrahedral $(a-\mathrm{Si}: \mathrm{H})$ and chalcogenide amorphous semiconductors. The atomic densities are representative. SRO, MRO, and LRO stand for short-, medium-, and long-range orders.

\begin{tabular}{|c|c|c|c|c|}
\hline \multirow{2}{*}{ Atomic density, $\mathrm{cm}^{-3}$} & \multirow{2}{*}{ Structural change } & \multicolumn{2}{|c|}{ Material } & \multirow{2}{*}{ Reversibility } \\
\hline & & $a-\mathrm{Si}: \mathrm{H}$ & Chalcogenide & \\
\hline$\sim 10^{22}$ & LRO & Photo-crystallization & $\begin{array}{l}\text { Photo-crystallization } \\
\text { Giant photo-contraction } \\
\text { Photo-polymerization } \\
\text { Photo-decomposition }\end{array}$ & Irreversible \\
\hline $\begin{array}{l}\sim 10^{20} \\
\sim 10^{18}\end{array}$ & $\begin{array}{c}\text { MRO } \\
\text { Defective SRO }\end{array}$ & Staebler-Wronski & $\begin{array}{l}\text { Photo-darkening } \\
\text { Photo-induced anisotropy } \\
\text { Photo-induced ESR }\end{array}$ & Reversible \\
\hline
\end{tabular}

ible and others are reversible, and, in general, irreversible phenomena exhibit greater structural changes. A well-known irreversible change is the photopolymerization phenomenon observed in as-evaporated $\mathrm{As}_{2} \mathrm{~S}_{3}$ films. ${ }^{7,8}$ On the other hand, at least, two reversible changes, i.e. the photodarkening and the photoinduced anisotropy, are known to exist. ${ }^{3,5,6,8}$ The details will be discussed in Sec. 3 .

\subsection{Discussion}

It is interesting to note in Table I that all the photoinduced phenomena involving atomic sites less or more than $\sim 10^{20} \mathrm{~cm}^{-3}, \sim 1 \%$ of the total atom density, are reversible and irreversible. It can be understood that the irreversible phenomena can involve greater atom numbers, since the changes occur toward more stable atomic structures (see Table I). Why, then, should the atomic sites be less than $1 \%$ in the reversible changes?

This fact can be accounted as follows: The density of $1 \%$ reads one photoinduced atomic site per cube with a side length of $5-6$ atoms, which is $1-3 \mathrm{~nm}$, depending on the atomic bonds involved, i.e., covalent and/or van der Waals bonds. This length implies that, in a reversible change, a photoinduced atomic configuration can be metastable, provided that produced strain is confined in this cube. It can then be relaxed into a stable structure with thermal relaxation induced by annealing. Alternatively, if more defective sites were generated in the cube, interaction between the defective sites would be stronger, and hence cooperative relaxation (annihilation) might occur toward stable atomic configurations. We can thus assume that the defective atomic density of $1 \%$ is the limit arising from the structures which can localize photoinduced strains. ${ }^{3)}$ It is interesting to note that the critical length of $1-3 \mathrm{~nm}$ is comparable to the medium- range structural length in chalcogenide glasses. ${ }^{16,17}$ We can envisage one photoinduced defect in a crystalline structure with a scale of $1-3 \mathrm{~nm}$.

Why, then, should the site density of the photoinduced dangling bonds be less than $10^{18} \mathrm{~cm}^{-3}$ ? The density may be related to the nature of nonequilibrium disordered structures. ${ }^{10}$

It may also be worthwhile to consider the photoinduced phenomena from a chemical point of view. We note in Table I that chalcogenide glasses exhibit a variety of photoinduced phenomena, while the tetrahedral material possesses a fewer. How can we understand these contrasting features?

The origin can be sought in the chemical bonding structures. As illustrated in Fig. 1, chalcogenide glasses are assumed to consist of covalent clusters held together with weak intermolecular forces of the van der Waals type. ${ }^{16}$ In other words, the glass possesses a dualistic bonding nature and $2 \leqslant Z \leqslant 3$, where $Z$ is the covalent coordination number averaged over the constituent atoms. ${ }^{16}$ This flexible structure with the low coordination number seems to cause many kinds of photoinduced phenomena. For instance, the photopolymerization mentioned in 2.1 can occur because there exist intra- and inter-molecular bonds. ${ }^{7,8}$ We will also see in the next section that the photodarkening can be attributed to the existence of strong and weak bonds. In contrast, the atomic bond involved in the tetrahedral material is of one kind, i.e., only covalent, and, accordingly, photoinduced changes are restricted in variety. Actually, Shimizu argues that the Staebler-Wronski effect could not occur if all Si atoms were tetrahedrally bonded with each other $(Z=4)$ (Ref. 5).

Here, it is tempting to consider whether photoinduced phenomena appear in amorphous rare-gas solids. The material contains only van der Waals bonds $(Z=0)$, and it may be very soft. ${ }^{16,21}$ Unfortunately, as we know; photoinduced 


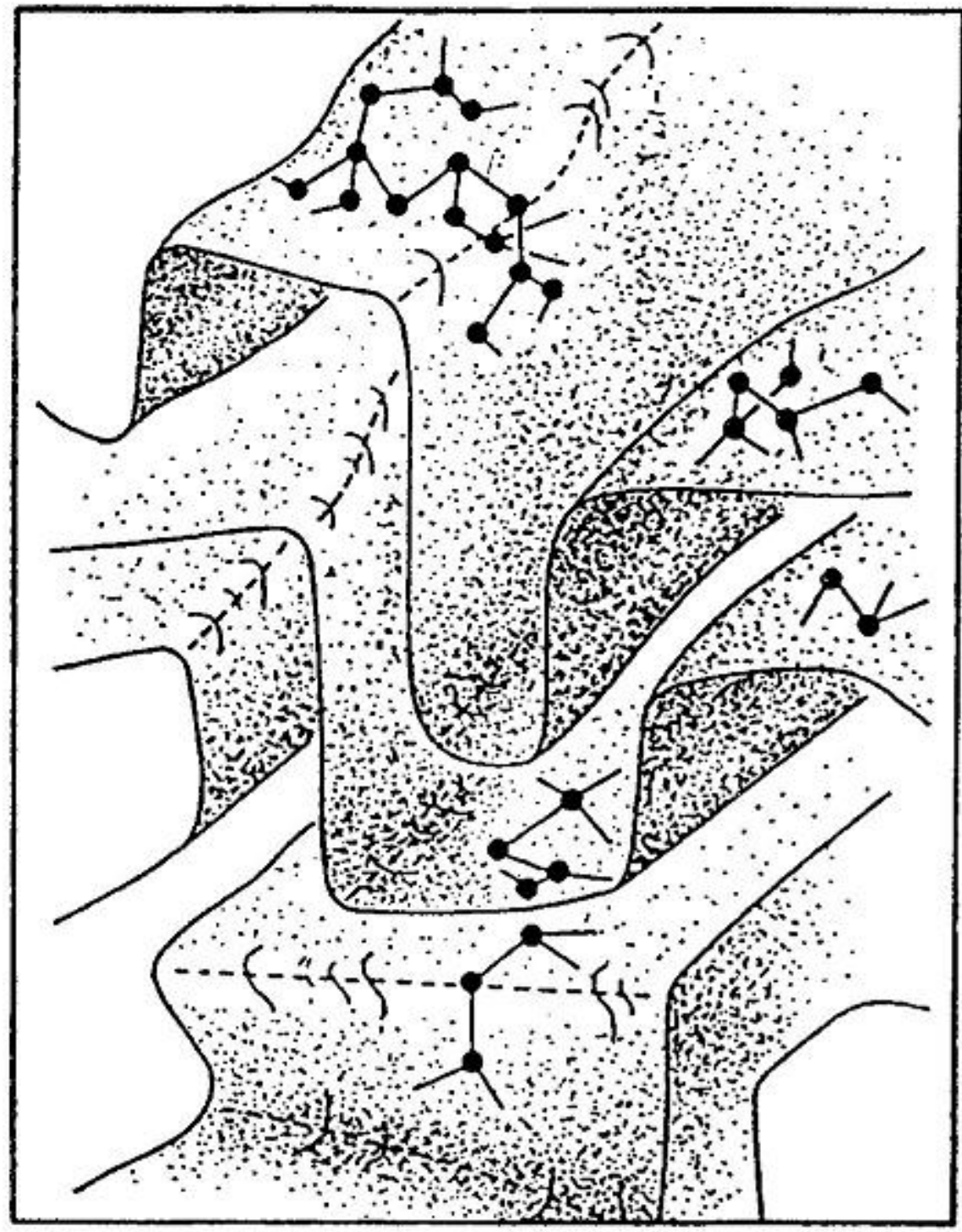

FIG. 1. A schematic illustration of a chalcogenide glass consisting of fourfold (Ge), threefold (As, Sb), and twofold ( $\mathrm{S}, \mathrm{Se}, \mathrm{Te}$ ) coordianted atoms.

phenomena in such materials, have not been studied so far. However, it is plausible that only photoinduced crystallization can occur, since the van der Waals bond is nondirectional and flexible. Phenomena similar to the StaeblerWronski effect cannot appear, since there exist no covalent bonds and local bistable configurations cannot be sustained.

Summarizing these considerations, we conclude that the existence of two kinds of bonds in chalcogenide glasses is responsible for a variety of photoinduced phenomena. In other words, the low $Z$ is essential. ${ }^{4)}$

\section{PHOTODARKENING AND RELATED STRUCTURAL CHANGES}

The photodarkening phenomenon observed in chalcogenide glasses has so far attracted considerable interest, and the structural changes have been studied extensively. Below we give a brief review of the photodarkening and related phenomena, and then consider some structural models proposed so far.

\subsection{Observations}

When a chalcogenide glass such as $\mathrm{As}_{2} \mathrm{~S}_{3}$, which has been annealed at $T_{g}$ in advance, is illuminated at room temperature, or at temperatures substantially lower than $T_{g}$, the sample exhibits reversible changes in volume, optical, electrical, photoelectrical, mechanical, chemical, and thermal properties. ${ }^{4-6,8}$ Here, the optical change includes the photodarkening, which refers to the feature that illuminated mate-

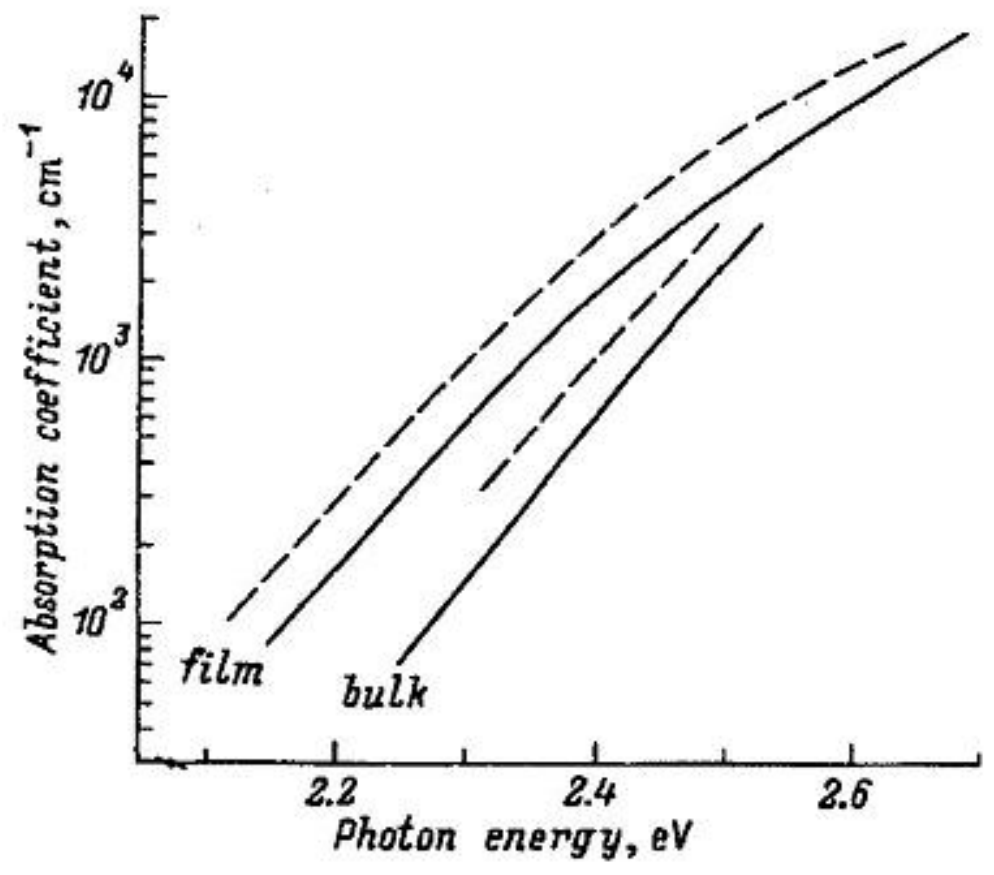

FIG. 2. Photodarkening in deposited and bulk $\mathrm{As}_{2} \mathrm{~S}_{3}$ at room temperature. Solid and dashed lines show annealed and iluminated states. The film char* acteristics are measured using the photothermal spectroscopy [K. Tanaka, Y. Ichimura, and K. Sindoh, J. Appl. Phys. 63, 1815 (1988)].

rials appear to be darkened. As shown in Fig. 2, this darkening is caused by a reduction of the optical bandgap energy. An increase in the refractive index $(\Delta n / n \simeq 0.03 / 2.6$ in $\mathrm{As}_{2} \mathrm{~S}_{3}$ ) accompanies this absorption change. The electrical change appears as an increase in the ac conductivity, ${ }^{5}$ while dc conductivity change cannot be examined, since the material is nearly insulating. ${ }^{17}$ As for the photoelectrical change, photoconductive degradation was found to occur as a result of the spectral change. ${ }^{5,22}$ It is not conclusive whether the hole mobility undergoes some modifications as a result of illumination. ${ }^{6.7}$ The mechanical, chemical, and thermal changes imply that the material becomes softer and unstable upon illumination. ${ }^{5}$ Also consistent with these rigidity changes is the volume expansion $\left(\Delta V / V=0.5 \%\right.$ in $\left.\mathrm{As}_{2} \mathrm{~S}_{3}\right)$ upon illumination. More surprisingly, Tanaka et al. have demonstrated giant volume expansion with sub-bandgap illumination, which can be utilized to produce microlenses. ${ }^{6}$ However, the expansion does not occur if a sample is illuminated under hydrostatic compression, despite the appearance of photodarkening. ${ }^{5}$ We assume therefore, that photoexpansion and photodarkening are not directly related. ${ }^{23}$

To obtain insight into microscopic structural changes accompanying the photodarkening, diffraction, extended $x$-ray absorption fine structure (EXAFS) and vibrational spectroscopic studies have been performed. ${ }^{3-8}$ However, it seems difficult to extract a unified model for the structural change from these studies, since the structure itself is controversial. ${ }^{16,17}$ In addition, as implied above, it is plausible that different kinds of structural changes are induced by illumination.

Diffraction studies using $\mathrm{x}$-rays and neutrons have been reported by some groups. ${ }^{3.5}$ Figure 3 shows an $\mathrm{x}$-ray result reported by the present author using bulk $\mathrm{As}_{2} \mathrm{~S}_{3}$ glass. ${ }^{23}$ Illumination and diffraction measurements have been performed at room temperature in situ, in order to exclude thermal expansion effects and to detect minute changes. We see that the $\mathrm{X}$-ray intensity difference (annealed-illuminated) is positive at about the first sharp diffraction peak (FSDP), 

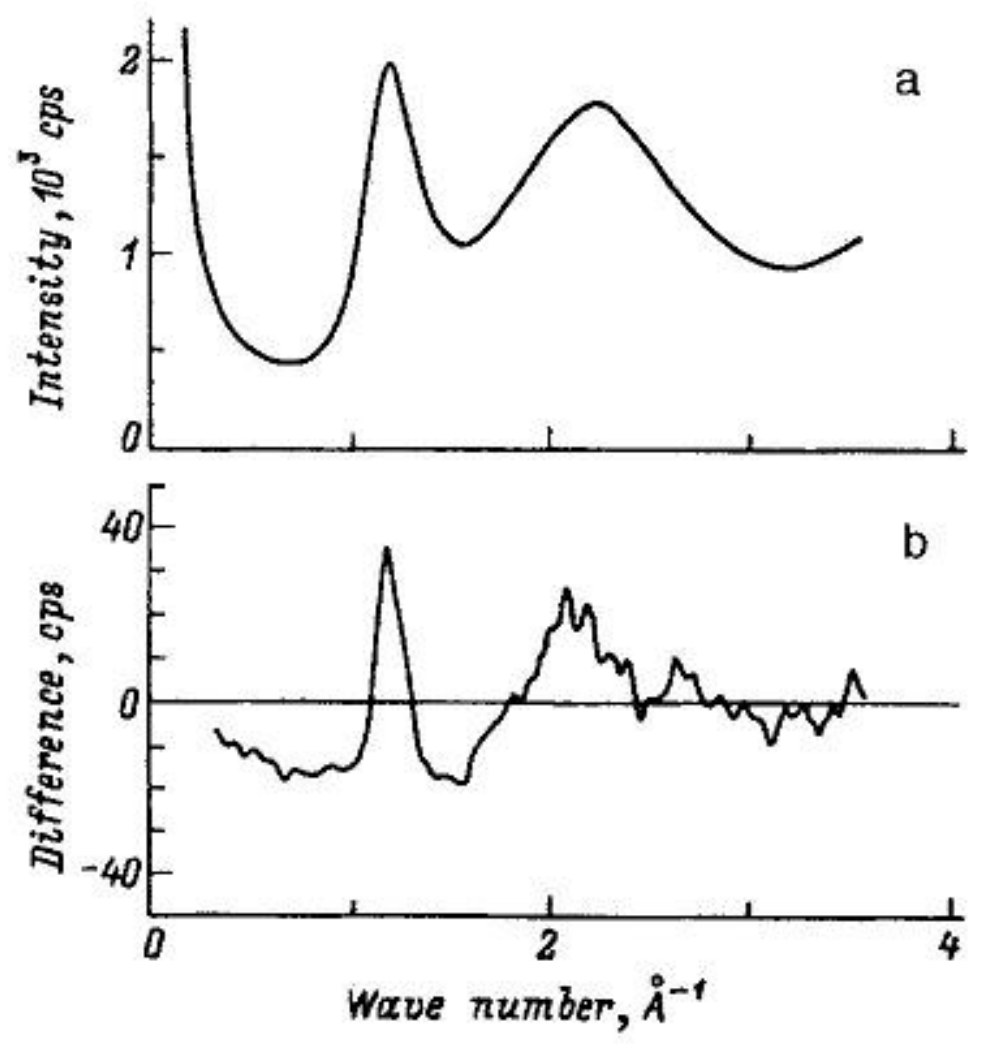

FIG. 3. X-ray diffraction patterns of an $\mathrm{As}_{2} \mathrm{~S}_{3}$ bulk glass before and after illumination. $a-$ shows the annealed state, and $b-$ shows the difference obtained by subtracting the $x$-ray intensity of the illuminated pattern from that of the annealed pattern.

which indicates photoinduced disordering in the mediumrange order. This experimental result has been confirmed by some researchers, ${ }^{3,5}$ while the interpretation of the FSDP remains controversial. ${ }^{16,17}$ The author prefers the so-called distorted-layer model ${ }^{16}$ while others use three-dimensional models, etc. ${ }^{17}$

Some EXAFS studies have been reported. ${ }^{3,5,24}$ However, the results reported for binary glasses are not necessarily consistent. ${ }^{3,5}$ Creation of homopolar bonds and fluctuation enhancement of As-S-As bond angles have been pointed out. Kolobov et al. report in situ investigation for amorphous $\mathrm{Se}^{24}$

Vibrational spectra have been obtained through Raman and infrared (IR) studies. ${ }^{4-8}$ In Raman scattering spectra of illuminated $\mathrm{As}_{2} \mathrm{~S}_{3}$ films, a trace of As-As bonds has been detected at $231 \mathrm{~cm}^{-1}$, and the intensity of the low-frequency Raman peak located at $\sim 25 \mathrm{~cm}^{-1}$, the so-called boson peak, is substantially reduced..$^{5,7}$ No marked changes in the As-S vibrational band centered at $340 \mathrm{~cm}^{-1}$ are reported. In contrast, in the IR spectroscopy, which may be more amenable to quantitative evaluation, slight broadening of the As-S vibrational band has been detected. ${ }^{4}$

\subsection{Discussion}

How can we understand these macroscopic and microscopic changes? Taking the reversible features into account, we can assume a configuration-coordinate diagram such as shown in Table I (Ref. 5), in which an illuminated state is shown as quasi-stable. Then, the problem turns to the structural element involved.

Here, as illustrated in Fig. 4, the structural models proposed so far can be classified into two groups. In all models, relatively ordered structures are assumed to be the annealed state. For the illuminated state, the defect models (a) and (b)

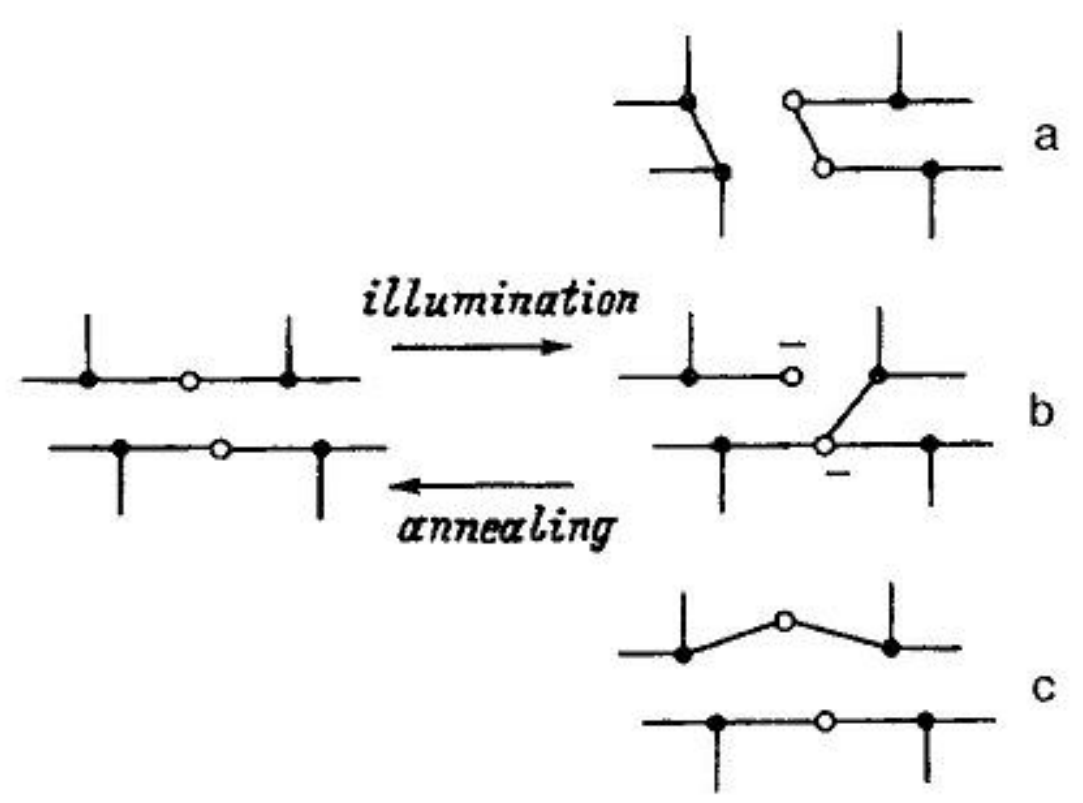

FIG. 4. Schematic illustration of structural models proposed for the photodarkening and related phenomena. $a$ and $b$ show defect models, and c represents distortion models.

presume creation of some kind of defects and, in contrast, the distortion models (c) postulate that randomness increases in normal bonding configurations.

\subsubsection{Defective structure models.}

Owen and others have emphasized through their Raman scattering measurements that As-As bonds are formed in illuminated states (Fig. 4a). ${ }^{5,7.8}$ It is theoretically predicted that As-As bonds provide electronic states in the bandgap, ${ }^{3,17}$ and hence the photodarkening may appear if the homopolar bonds are created. Nonetheless, the model cannot be a universal explanation of the photoinduced change, since the photodarkening appears in elemental materials, $S$ and $\mathrm{Se}^{5}$ In addition, no evidence of photoinduced $\mathrm{Ge}-\mathrm{Ge}$ bonds is obtained throught Raman studies. ${ }^{23}$

Street and others have developed defective models using the charged-defect concept which was originaliy proposed by Mott. ${ }^{5}$ The charged defects are assumed to produce electronic states in the band-tail regions, ${ }^{17}$ and hence the bandgap is reduced upon illumination. These defects with the density of $\sim 1 \%$ would manifest specific vibrational peaks, which were investigated by Kolobov et al. ${ }^{25}$

\subsubsection{Distorted structure models.}

In the last model illustrated in Fig. 4, some kind of photoinduced distortions in normal bonding configurations are assumed. Since modifications of covalent bond lengths requires substantial energy, and since the strained structures may not be quasi-stable, such distortions cannot be envisaged in the reversible photodarkening.

Plausible structural changes can then be sought in the distortions in bond angles, dihedral angles, and van der Waals distances. For instance, Utsugi and Mizushima emphasize the angular distortion in the short-range order, on the basis of their IR studies. ${ }^{4}$ Angular fluctuation will increase the width of the conduction band, since the conduction band in chalcogenide glasses consists of the antibonding state of 
covalent bonds. ${ }^{16,17}$ As a result, the photodarkening can appear.

On the other hand, distortions in the dihedral angle and the van der Waals distance enhance the randomness in the medium-range structural order. Pfeiffer et al., assume distortion of dihedral angles on the basis of their EXAFS studies. ${ }^{3}$ In contrast, the present author has proposed the intermolecular distortion through bond-twisting motion of chalcogen atom. ${ }^{5}$ These two kinds of structural changes appear to be consistent with the FSDP weakening and broadening, shown in Fig. 3. The structural changes will modify the width of the valence band, since the width of the valence band is strongly influenced by the interaction between lone-pair $p$-orbital electrons of chalcogen atoms. ${ }^{16,17}$

At present, it difficult to determine which structural change is the most responsible. We expect that further insight can be provided if it is disclosed that photodarkening is caused by the changes in the bottom of the conduction band and/or in the top of the valence band. However, the accuracy of the photoelectron spectroscopy, which is capable to determine the position of the band edges, is not sufficient to resolve a shift of $\sim 50 \mathrm{meV}$. Alternatively, we can assume that the three structural changes are interrelated, since the structural relaxation is characteristic of chalcogenide glasses that possess low $Z$. In other words, if an intermolecular bond is distorted upon illumination, structural relaxation will necessarily occur, which would lead to appreciable angular distortions.

\subsubsection{Miscellaneous.}

When dealing with the photodarkening in $\mathrm{As}_{2} \mathrm{~S}_{3}$ and similar materials, we should note characteristic differences and similarities between the bulk and the annealed films. In general, structures of the bulk and the annealed films are assumed to be similar, while it has been demonstrated that the annealed film still contains an appreciable number $(\sim 1 \%)$ of As-As bonds. ${ }^{7,8}$ In fact, Fig. 2 shows that the absorption edges of an annealed film and the corresponding bulk are clearly different. Wrong bonds (homopolar bonds in stoichiometric compounds) seem to cause the difference. Surprisingly, however, the photoinduced red-shifts are nearly the same, $\sim 50 \mathrm{meV}$, in both materials.

We should also note that when $\mathrm{As}_{2} \mathrm{~S}_{3}$ is exposed to linearly polarized light, the photodarkening and the photoinduced anisotropy appear simultaneously. The mechanisms of these phenomena were found to be different. ${ }^{5,26,27}$ Accordingly, it is clear that at least two kinds of structural changes are induced upon illumination. Further studies are needed in order to obtain one-to-one correspondences between macroscopic changes and microscopic structural changes, in which the latter is largely speculative.

\section{SUMMARY}

Overall features of the photoinduced phenomena observed in amorphous semiconductors are considered from two aspects. From the atomic density, it appears that the reversible phenomena cannot accompany structural changes at atomic sites more than $\sim 1 \%$ of the total atom density. From a chemical point of view, the reason why chalcogenide glasses exhibit a variety of phenomena can be sought in the dualistic bonding nature which involves covalent and van der Waals bonds.

The photodarkening and related phenomena are critically reviewed. Many macroscopic features have been revealed, while the mechanism remains speculative. This is because the structural changes appear to extend to medium-range scales, for which no convincing experimental tools are yet available. In contrast, the phenomena appear to be promising for fabrication of functional devices. ${ }^{5,6}$

This article is dedicated to Professor Kolomiets, who is undoubtedly the founder of amorphous semiconductor physics. He also brought up many able scientists such as Professor Lyubin and Dr. Kolobov. I cannot forget his strength and warmth at the Ioffe Institute in 1987.

\footnotetext{
1) The phase-change process can be reversible with thermal annealing and quenching, which are induced with pulsed illumination at different light intensities and different pulse widths. In the reversible photoamorphization of crystalline $\mathrm{As}_{50} \mathrm{Se}_{50}$ films, substrates are responsible for the peculiar phenomenon.

${ }^{2)}$ Breaking of covalent bonds and reptile motion of chain fragments are probably needed, like the crystallization process in chain polymers (see Ref. 18).

${ }^{3)}$ In order to confirm this speculation, however, lattice dynamical calculation in large amorphous networks is needed.

4) In this sense, photoinduced phenomema can appear also in oxide glasses, as they do. ${ }^{5}$ Nonetheless, the atomic bond is more ionic, and the dualistic bonding nature is not prominent. Accordingly, they exhibit fewer kinds of photoinduced phenomena, like $a-\mathrm{Si}: \mathrm{H}$.
}

'N. F. Mott and R. W. Gurney, Electronic Processes in Ionic Crystals (Dover, N.Y., 1964).

${ }^{2}$ A. V. Kolobov and S. R. Elliott, Adv. Phys. 40, 625 (1991).

${ }^{3}$ G. P. Pfeiffer, M. A. Paesler, and S. C. Agarwal, J. Non-Cryst. Solids 130, 111 (1991).

${ }^{4}$ Y. Utsugi and Y. Mizushima, Jpn. J. Appl. Phys. 31, 3922 (1992).

${ }^{5}$ K. Shimakawa, A. Kolobov, and S. R. Elliott, Adv. Phys. 44, 475 (1995).

${ }^{6} \mathrm{~K}$. Tanaka, Current Opinion in Sol. St. and Mater. Sci. 1, 567 (1996).

${ }^{7}$ V. I. Mikla, J. Phys.: Condens. Matter 8, 429 (1996).

${ }^{8}$ M. Fnumar, M. Vlcek, Z. Cemosek, Z. Polak, and T. Wagner, J. Non-Cryst. Solids 213\&214, 215 (1997).

${ }^{9}$ G. Dale, A. E. Owen, and P. J. S. Ewen, in Physics and Applications of Non-Crystalline Semiconductors in Optoelectronics, edited by A. Andriesh and M. Bertolotti (Kluwer, Dortrecht, 1997) p. 45.

${ }^{10}$ K. Tanaka, J. Non-Cryst. Solids 139, 179 (1992).

"M.-C. Lee, C.-J. Tseng, C.-R. Huang, and T.-H. Huang, Jpn. J. Appl. Phys. 26, 193 (1987).

${ }^{12} \mathrm{~T}$. Ohta, N. Akahira, S. Ohara, and I. Satoh, Opto-Electron. 10, 361 (1995).

${ }^{13}$ M. Nakamura, Y. Wang, O. Matsuda, K. Inoue, and K. Murase, J. Non-Cryst. Solids 198-200, 740 (1996).

${ }^{14}$ J. Solis, C. N. Afonso, S. C. W. Hyde, N. P. Barry, and P. M. W. French, Phys. Rev. Lett. 76, 2519 (1996).

${ }^{15}$ A. V. Kolobov and S. R. Elliott, J. Non-Cryst. Solids 189, 297 (1995) [sic].

${ }^{16} \mathrm{R}$. Zallen, The Physics of Amorphous Solids (John Wiley \& Sons, N.Y., 1983).

${ }^{17}$ S. R. Elliott, Materials Science and Technology, edited by Zarzycki (VCH, Weinheim, 1991). Vol. 9, p. 375.

${ }^{18}$ T. P. Russell et al., Nature (London) 365, 235 (1993).

${ }^{19}$ H. Fritzsche, Solid State Commun. 94, 953 (1995). 
${ }^{20}$ K. Shimizu, T. Shiba, T. Tabuchi, and H. Okamoto, Jpn. J. Appl, Phys. 36, 29 (1997).

${ }^{21}$ A. Kouchi and T. Kuroda, Jpn. J. Appl. Phys. 29, L807 (1990).

${ }^{22}$ N. Toyosawa and K. Tanaka, Phys. Rev. B 56, 7416 (1997).

${ }^{23}$ K. Tanaka, Phys. Rev. B (in press).
${ }^{24}$ A. V. Kolobov, H. Oyanagi, Ke. Tanaka, and K. Tanaka, Phys. Rev. B 55, 726 (1997).

${ }^{25}$ A. V. Kolobov et al., (in press).

${ }^{26}$ K. Tanaka, K. Ishida, and N. Yoshida, Phys. Rev. B 54, 9190 (1996).

${ }^{27}$ K. Ishida and K. Tanaka, Phys. Rev. B 56, 206 (1997). 